\title{
LA MORT A LE VISAGE D'UNE FEMME. À PROPOS DES HISTOIRES SINGULIÈRES DE JEAN MUNO
}

\author{
Renata Bizek-Tatara
}

\begin{abstract}
In his collection of phantasy short stories Les histoires singulières, Muno breaches the convention of the genre that concerns the presentation of death and phantoms. Frightening and fascinating at the same time, death always assumes the figure of a woman who is either average or seducing. Her coming is announced by female messengers. Death in Muno is not a tragic event: it is a promise of a better life and a salvation from the worries of schematic and overwhelming everyday reality.

Keywords : Jean Muno; phantasy; Histoires singulières; death; woman.

Résumé : Dans son recueil de nouvelles fantastiques Les histoires singulières, Jean Muno brise le carcan qui fige habituellement la représentation de la mort et des revenants. A la fois effrayante et fascinante, la mort prend toujours l'apparence d'une femme, ordinaire ou séduisante, et est annoncée par des acolytes insolites, tous aussi de sexe féminin. La fin chez Muno n'est pas un événement tragique : elle est la promesse d'une existence meilleure, une vraie délivrance des tracas d'un quotidien schématique et étouffant.
\end{abstract}

Mots-clés : Jean Muno ; fantastique ; Histoires singulières ; mort ; femme.

Le fantastique de Jean Muno se distingue d'emblée de celui des grands maîtres du genre, tels que Jean Ray et Thomas Owen : « loin d'effrayer ou d'égarer l'esprit, il se fait plausible et, pour tout dire, sécurisant ». (Denis 1989: 77) L'écrivain fait éclore l'insolite au sein du quotidien, profondément routinier, fade et banal. Ainsi, son fantastique, ancré toujours dans une réalité solide et familière, en constitue nettement une prolongation. Muno le confirme : " Je crois cerner la vie de plus près grâce à ces déformations qu'on peut qualifier de fantastiques. C'est une façon pour moi d'exprimer mieux la réalité, de l'exprimer comme on exprime un jus de citron ». (Muno 1989 : 46) Il ne s'agit donc pas d'un jeu littéraire gratuit. Le fantastique munolien touche aux questions essentielles : il met en relief des obsessions et angoisses humaines dont la plus forte est celle de la mort. $C^{\prime}$ est pourquoi ses histoires, quoique point effrayantes, engendrent une impression de malaise et de désespérance accablante. ${ }^{1}$

\footnotetext{
1 Par là Muno réalise l'un des principes constitutifs de la théorie du fantastique proposée par T. Todorov. Celuici dénie la peur en tant que critère distinctif du fantastique, en démontrant qu'ainsi le genre dépendrait du sang-froid du lecteur. Selon Todorov, l'intention fondamentale du récit fantastique réside dans sa capacité de créer une situation de malaise et de faire surgir une sensation d'inquiétude face à un " dérèglement » de ce qui apparaissait si bien réglé. Nous voulons pourtant souligner que l'hésitation, qui dans la théorie todoro-
} 
Nous voulons privilégier un puissant motif de sa création littéraire, celui de la mort, qui marque foncièrement le recueil de nouvelles fantastiques Les histoires singulières. Son image et sa fonction, visiblement différentes de celles que nous offrent d'autres auteurs fantastiques, font preuve d'une grande originalité de l'écrivain dans l'inventivité.

La mort est présente dans presque toutes les nouvelles ${ }^{2}$ et elle reste, avec tout ce qui l'accompagne, leur thème majeur. Son spectre rôde dans l'univers fantastique de Muno, hante ses personnages et leur fait découvrir une autre réalité, parallèle à la leur, un monde insolite de l'au-delà. Presque tous les récits évoquent la rencontre de l'homme avec des revenants des ténèbres ou avec sa propre mort, personnifiée ou invisible, qui est à la fois affrontement et union, fin et début, peine et délivrance.

Les morts-vivants, autour desquels s'organisent maints récits fantastiques, abondent largement dans l'univers fictionnel de Jean Muno, mais la particularité de ses êtres prodiges consiste dans le fait qu'ils sont presque toujours de sexe féminin : goules, "revenantes » et "vampiresses », privées d'allure monstrueuse, apparaissent soit foncièrement ordinaires soit séduisantes et, tout en restant porteuses de la mort, bienveillantes envers les mortels. ${ }^{3}$

Dans La dame-au-chien, le protagoniste côtoie, lors de ses promenades quotidiennes, le fantôme d'une femme, sans même se rendre compte de la nature insolite de celle-ci. Elle a l'air tout à fait commun : c'est « la soixantaine robuste», au « large visage un peu camus de paysanne rosi par la promenade » et aux « yeux clairs » dévisageant l'homme avec une intensité dérangeante. (Muno 1979: 36) «Quel que soit le temps, elle porte de courtes bottes en caoutchouc et un ample ciré vert-de-gris, en pèlerine, qui chuinte comme une armure souple ». (Muno 1979: 26) Remarquons que ce n'est pas l'aspect extérieur de la femme qui éveille la curiosité du personnage, mais son comportement schématique et inlassablement identique : chaque jour, à trois heures dix-huit, elle surgit du même endroit et, avec la même expression de visage, arpente le même chemin et disparaît comme la brume à la sortie du parc. «Une rencontre unique, obsédante comme une idée fixe ». (Muno 1979: 36)

L'homme ne découvre l'identité de la promeneuse " qui ne respire pas » qu'au ha$\operatorname{sard}^{4}$, mais il n'éprouve pas de crainte. Il est plutôt ému de pouvoir participer à un tel mystère : « La mort ne me fait pas peur, elle n'est qu'une attente. Un jour, je ne prendrai pas la peine de retourner [...] ». (Muno 1979: 39) Le protagoniste semble se trouver à la frontière qui sépare la vie de la mort et, finalement, il ne sait plus s'il est « vivant ou ... comme Elle, la dame-au-chien ». (Muno 1979 : 39) Dans cette nouvelle, Muno renverse visiblement les rôles traditionnellement prescrits aux êtres surnaturels et prend le

vienne conditionne la littérature fantastique, est complètement absente des récits de Muno: ses personnages, confrontés à l'insolite, l'acceptent d'emblée en tant que tel et n'en éprouvent pas l' « horreur surnaturelle » au sens où l'entend F. H. Lovecraft. (Voir : Todorov $1970: 28,45$ )

2 Le thème de la mort n'apparaît seulement pas dans la nouvelle Bande dessinée.

3 Dans le fantastique traditionnel, le revenant est en général présenté en tant que maléfique et anxiogène. "Lié à un sort malheureux et tragique, constate Ch. Grivel, il est issu d'un mort, mal mort et mal enterré, injustement frappé, dont le sang crie la vengeance ; il signe un trouble, dénote un dérangement profond ». (Grivel $1983: 36)$

4 Le jour de la Toussain, lors de la visite habituelle au cimetière, le héros jette furtivement un coup d'œil sur une tombe abandonnée et éprouve un choc : dans le médaillon de la stèle mortuaire, il reconnaît la dame du parc, décédée depuis une trentaine d'années, qui le regarde comme au moment où ils se croisaient. La découverte insolite le pousse à venir au parc le lendemain. À trois heures dix-huit, le spectre apparaît comme d'habitude et s'assoit sur le banc, à côté de lui. 
contre-pied des habitudes admises. Une convention bien établie du fantastique veut que les revenants fassent horreur aux vivants et que ceux-ci s'enfuient. ${ }^{5}$ Or, dans La dame-auchien, c'est de toute évidence l'homme qui, attiré par l'Inconnu, cherche la compagnie de la femme ténébreuse.

Muno rompt aussi avec les conventions du genre dans La voix du sang. Cette nouvelle peut même être considérée comme une parodie du thème fantastique traditionnel $\mathrm{du}$ vampirisme. L'auteur y met en scène une "vampiresse » d'origine moldave, au nom évocateur de Rita Bathory, ${ }^{6}$ qui tisse des liens amicaux avec ses victimes pour pouvoir se régénérer de leur sang sans scandale. Cette femme généreuse, poussée par "la soif d'autrui ", porte secours aux gens moralement désemparés et excelle à les revigorer, à les rendre heureux. Et en fin de compte, c'est elle qui devient en quelque sorte leur victime, car ce sont eux qui se nourrissent de son temps, de son attention et de son énergie. ${ }^{7}$ Les rôles sont donc inversés : celle dont la nature est par excellence démoniaque agit d'une façon inhabituelle des autres monstres; par contre ses victimes, par leur cannibalisme social, ressemblent davantage aux vampires que leur protectrice insolite.

Muno remet visiblement en cause la nature maléfique du vampire en lui faisant perdre son statut de monstre sanguinaire, figure du mal, allégorie fantastique du tueur en série. La tradition populaire considère le vampirisme comme un acte de haine et de vengeance, chez Muno la succion du sang n'est par contre qu'une nécessité. Son héroïne est tourmentée par l'idée de tuer et ressent sa condition comme une malédiction. Ainsi, elle inspire davantage de pitié que de terreur.

Notons aussi que le physique de la femme vampire n'est pas non plus conforme au clichés, car il est peu surnature ${ }^{8}$ : Rita a des canines un peu longues, un sourire qualifié de félin ou de tartare, d'étranges petites oreilles de chat et « un beau regard, intense et froid, de nyctalope ». (Muno 1979 : 50) Bien qu'elle ne soit pas aussi belle que Clarimonde de Gautier ou Carmilla de Sheridan le Fanu, elle apparaît tout de même attrayante : « Ni laide ni jolie. Sûrement pas banale. Le corps est assez anguleux, mais le visage a beaucoup de caractère même, [...] un certain charme. [...] Elle possède des yeux exceptionnels, tantôt gris et comme voilés, tantôt d'un bleu très pur, métallique ». (Muno 1979: 50) En mettant en scène la femme vampire qui ne se distingue par rien de particulier des mortels et en la dotant d'une nature bienveillante, Muno ne se plie pas aux lois du genre. Il supprime entièrement le côté angoissant de la créature insolite, (quoique elle reste toujours mortifère) et ainsi il atténue, dans une grande mesure, l'aspect terrifiant de la mort que donne la « vampiresse».

La mort revêt un visage également féminin dans Le gant de volupté. Au début, elle prend la forme d'un « gant de peau noire, à longue manchette évasée, une élégante main

\footnotetext{
Nous pensons ici aux théories de R. Caillois, P. H. Lovecraft, P. Penzoldt et L. Vax qui insistent sur le sentiment de la peur que l'éclosion du fantastique doit provoquer.

6 La comtesse Báthory, dite « la Comtesse sanglante » est une figure historique qui a contribué le plus à la fortune du mythe du vampire féminin. Pour garder sa beauté, elle prenait des bains du sang des vierges. Son carnet atteste l'effroyable chiffre de 610 meurtres. Arrêtée et emmurée vive en 1611, elle est morte en 1614, « sans croix et sans lumière ». (Tritter $2010: 64$ )

7 Pour Muno, les vampires sont plus quotidiens qu'insolites : « Ils sont quotidiens les vampires! Ils sont quelconques : des employés, des types inscrits à la sécurité sociale. Je n'ai pas eu la chance de rencontrer le comte Dracula, moi ! je ne peux que témoigner que des vampires bourgeois que j'ai côtoyés ». (Voir : Andriat 1989: 48)

8 Dans le fantastique canonique, les héroïnes vampires possèdent des traits humains d'extrême beauté et cruauté ; de plus, leur teint est d'une pâleur lunaire.
} 
de femme posée » qui imprègne l'air d'un léger parfum de femme, « entêtant, poivré, un peu pervers ». (Muno $1979: 16$ ) Ce petit bout de tissu, une synecdoque de la femme inconnue, acquiert, comme la main éponyme de Maupassant, une existence indépendante et offre au protagoniste des plaisirs sublimes : elle l'enivre de caresses et de baisers, lors de leurs étreintes amoureuses interminables. L'objet conserve les facultés de la main vivante de l'amante rêvée qui, devenant de plus en plus exigeante et possessive, rend la vie de l'homme impossible. Celui-ci consent donc à s'en débarrasser, comme d'une vieille chose inutile. Sa lâcheté, son ingratitude et le retour vers son épouse sont pourtant sévèrement châtiés.

$C^{\prime}$ est le sentiment de culpabilité qui conduit le protagoniste vers la mort : "sans peur ni volonté », « comme soulevé de l'intérieur par un intense désir de soumission », il cherche lui-même la propriétaire du gant pour qu'elle le punisse de sa malhonnêteté. Il la trouve telle qu'il se l'imaginait, belle et distinguée :

Immobile comme une réponse définitive, sa haute et mince silhouette émergeant de la brume. LA REPONSE. Elle l'attendait, il ne pouvait pas le reconnaître. La longue robe noire, si évidemment hors de saison, l'étroit fuseau luisant de pluie, la créature de frimas sortie du fond de sa mémoire, et le geste surtout, les bras repliés sur la poitrine, les mains croisées à la naissance du cou, cette attitude de chrysalide, cet hiératisme de cauchemar... Mais le visage [...] ou plutôt l'absence de visage, le regard sans paupières. (Muno 1979: 28)

Un humain avilit une femme insolite en rejetant son amour et il en subit de graves conséquences : la morte-vivante lui sectionne son poignet droit et provoque sa fin. Il ne faut cependant pas voir dans la violence de la créature une simple vengeance cruelle. C'est le désir obsessionnel du plaisir qui mène inéluctablement l'homme à sa perte. Dès le moment où la passion envahit Peter, il est complètement privé du pouvoir qu'il a sur lui-même : il se laisse docilement engager dans une liaison amoureuse interdite, presque malsaine, et ne se maîtrise plus. Muno insiste ici visiblement sur le châtiment qui suit la moindre faute et sur la faiblesse de l'homme qui devient l'artisan de son propre malheur.

Il en est de même dans $\mathrm{La}$ goule. La nouvelle exploite le thème d'une passion destructive d'une femme insolite et d'un mortel passif, la terrible antithèse freudienne ErosThanatos, souvent mise en exergue par la littérature fantastique. Muno y présente un homme traversant la frontière du sommeil et qui est pris dans le tourbillon d'un cauchemar. Le protagoniste se trouve sur une plage et voit sortir de l'océan une morte-vivante qu'il appelle l'infirme, car la moitié de son corps est paralysée, comme morte. Elle a un « visage inaltérable de poupée [...], un visage dont aucune expression ne vient troubler l'harmonie », au regard «étrange, intense et froid ». (Muno 1979: 117) La créature ténébreuse est pourtant attrayante : "belle comme le masque d'or de la momie, équivoque comme l'enveloppe parfaite d'un être disparu », elle a la " peau d'une délicatesse immatérielle " et une «jolie main de squelette ». (Muno 1979: 117) Notons que, malgré son physique cadavérique, l'infirme apparaît aux yeux du narrateur comme un être frêle, délicat et fascinant. Et bien qu'elle se métamorphose soudain en goule et, accompagnée d'Erinyes, conduise le héros vers la mort, celui-ci ne s'y oppose pas, ensorcelé par le charme de la douce « vampiresse ».

Cette histoire cauchemardesque, que Muno lui-même avoue ne pas comprendre (Muno 1986 : 54), contient la symbolisation de relations sexuelles problématiques entre le héros et l'infirme. Dès le début du texte, le comportement de celui-ci trahit la crainte de franchir la frontière corporelle de l'autre, figurée par une bouche d'ombre ouverte dans une 
paroi rocheuse. Hésitant et incertain, il éprouve « un sentiment d'irrémédiable » (Muno 1979 : 116) et il s'en sent, par conséquent, « perdu, avili et coupable ». (Muno 1979: 120) Ces sentiments suggèrent qu'il a commis une faute et qu'il doit en être châtié, ce que la présence des Erinyes, les déesses vengeresses, illustre à son tour'. L'homme meurt d'une agression sexuelle pour ne pas avoir assumé ses responsabilités dans les relations intimes : "Ses reins fléchissent, ses genoux plient... Il s'abandonne à l'étreinte inexorable, à la dévoration monstrueuse et douce. Au supplice, au viol ». (Muno 1979 : 121)

La définition de la goule, proposée par Le dictionnaire de symboles de J. Chevalier et d'A. Gheerbrant, confirme notre interprétation. Cet être insolite amalgame les traits caractéristiques de maintes entités divines ou infernales, notamment ceux de la sirène, qui n'est pas nécessairement une femme-poisson : elle apparait également sous la forme mifemme et mi-oiseau, comme la goule munolienne. L'infirme se transforme en aigle, oiseau de proie exprimant une « volonté de puissance inflexible et dévorante ». (Chevalier, Gheerbrant 1969 : 703) De plus, sa voix, comme celle des sirènes mythologiques, est une arme redoutable : son cri attire irrésistiblement le héros, le séduit et le conduit ensuite à la mort. Quant à la faillite sexuelle, elle est également liée au symbolisme de la sirène : ces femmes "perverses", " sacrificatrices des mâles ", absorbant "la quintessence de sang par osmose au cours de l'acte sexuel », interviennent « dans le sommeil de leur victime par des rêves érotiques épuisants ». (Bernard 1971 : 169)

Le héros de La goule est le Prométhée de son malheur et anticipe sa propre mort. Il comprend au début de l'histoire («Ce sera la fin », Muno 1979 :119) qu'il quittera sous peu le monde : il le sait « depuis le cri, depuis le premier cri, autrefois, dans le canyon de Provence » (Muno 1979: 121). Ensorcelé par la mort, l'homme suit humblement l'appel de celle-ci et va vers la mer, vers l'Inconnu, fasciné et terrorisé à la fois. (Muno 1979 : 120)

Dans Le mal du pays, la mort est également en connivence avec une femme. Blanche, la concierge, n'est «blanche que de prénom [...] Grise pour le reste, d'un gris sans rémission, comme les eaux du fleuve ». (Muno 1979 : 9) Elle est maîtresse d'un savoir étendu, ses paroles et ses gestes sont fort lourds de signification : le jour de l'anniversaire de la mort future du héros, aux dires du narrateur, elle se passe le doigt sur le front, comme si elle se signait devant un mourrant. Elle essaye de lui annoncer quelque chose, mais renonce confuse, en disant que ce n'est pas important. Son comportement étrange pousse pourtant Walter à courir, à fuir son quotidien étouffant, à chercher l'oubli dans l'Inconnu. Il sent instinctivement que quelque chose va se passer, que cette nuit, il lui faut « chercher ailleurs, marcher au hasard, assez longtemps, pour ne plus entendre la voix obsédante » de la concierge (Muno 1979 : 11). Son errance le mène jusqu'à une digue au bout de laquelle, dans « le pays natal » (qui est une métaphore de la mort chez Muno), il y a un autre monde. Walter n' est pas encore prêt à y faire face, « de la peur de se perdre et de désobéir » et renonce à y pénétrer. (Muno 1979:12) Il attendra encore trois ans pour passer la frontière, rejoindre la mer, l'obscur royaume souterrain de la mort.

De toute évidence, la Concierge, dont le physique montre une affinité avec l'eau grise, ${ }^{10}$ est dépositaire d'un savoir surnaturel. Il en est de même dans L'iguane, où Eva

\footnotetext{
9 Les Erinyes sont considérées comme les instruments de la vengeance divine, à la suite des fautes des hommes, qu'elles poursuivent en semant l'épouvante dans leur cœur. Dans l'Antiquité, on les identifiait à la conscience. Intériorisées, elles symbolisent le remords, le sentiment de culpabilité, l'autodestruction de celui qui s'abandonne au sentiment d'une faute commise. Voir : (Chevalier, Gheerbrant 1969:411,412)

${ }^{10}$ Nous voulons souligner que chez Muno, comme chez Bachelard, l'eau et la mort constituent deux éléments symbiotiques.
} 
mystérieuse, une femme des origines, mystique, fatale et éternelle, révèle au héros le secret recherché. C'est elle qui initie Dolf Cnorrhinmus dans la voie vers l'Inconnu : Eva le mène sur l'Achéron, fleuve qui, dans la mythologie, sépare le rivage des vivants de celui des morts, et l'aide à exorciser la mort, figurée par l'araignée. ${ }^{11}$ Enfin, délivré de ses obsessions et de ses angoisses, l'homme commence un long voyage, " un retour aux sources $»^{12}$, pareil à celui des saumons au moment du frai, "voyage paradoxal », comme le remarque $\mathrm{F}$. Thyrion, " puisqu'il mène aussi à la mort vers laquelle tout converge ». (Thyrion 1989 : 75) Dans cette nouvelle, l'émissaire des ténèbres est une sorte d'initiatrice qui entraîne l'homme vers l'Inconnu, vers une nouvelle vie, devenant à la fois l'origine et le point de chute, celle qui donne la vie et la mort. ${ }^{13}$

Il est intéressant d'observer que la mort, à la fois effrayante et fascinante, a toujours son acolyte, une femme énigmatique, qui est munie de mêmes traits antithétiques. Bien que celle-ci soit douée d'une puissance mortifère remarquable (elle est responsable de la déchéance de l'homme), elle attire visiblement les mortels : les héros désirent l'approcher et se confient humblement à elle, corps et âme. Ce choix des femmes pour les êtres fantastiques bénins renvoie au fameux mythe du « sexe faible » auquel Muno semble recourir. Par son anatomie, la femme incarne la beauté, la finesse et la délicatesse qui inspirent généralement la confiance, la sympathie ou l'admiration. Dans cette optique, il n'est pas étonnant que les créatures insolites munoliennes suscitent, par seuls leur sexe et les traits lui associés, les mêmes sentiments positifs.

Il y a encore un autre élément qui rend les mortes-vivantes attrayantes : c'est le parfum qu'elles exhalent, agréable et subtil. Dans La dame-au-chien, l'odeur de la boue et de la menthe mêlées marque le passage de la défunte et flotte au-dessus de sa tombe. La parution du gant mystérieux, dans Le gant de volupté, est également suivie d'un léger parfum de femme : " une odeur entêtante, poivrée, un peu perverse, celle-là qui, très estompée, flottait dans le café avec la musique douce ». (Muno $1979: 18$ ) Le fait d'attribuer aux créatures d'outre-tombe une douce saveur accentue indubitablement leur charme et met en avant leur féminité.

Muno brise donc le carcan qui fige, la plupart du temps, la représentation de la mort et des revenants. Dans ses textes, la fin n'est pas un événement tragique et traumatisant, elle est la promesse d'une existence meilleure, une vraie délivrance des tracas d'un quotidien morne, schématique et étouffant. La vie terrestre acquiert en revanche une valeur négative, car elle ne constitue qu'une suite d'échecs et de déceptions ${ }^{14}$ : elle est l'attente pénible d'une nouvelle vie que ses héros espèrent goûter au delà du monde réel. C'est pourquoi ils tentent de passer, malgré la crainte de l’Inconnu, de leur univers précis et familier à un monde mystérieux d'outre-tombe ${ }^{15}$.

$\overline{11}$ Dans l'esthétique fantastique, l'araignée est intrinsèquement identifiée à la femme, probablement à cause du rituel de séduction et d'accouplement de la « veuve noire ».

${ }^{12}$ Encore une fois, la quête de l'enfance croise l'aspiration à la mort.

${ }^{13}$ Muno confirme dans La maison natale: «Peut-être qu'il faut se garder de revenir dans sa maison natale [...], qu'étant celle du jour qui vous a vu naître, elle l'est aussi de la nuit d'où vous êtes issu ? Peut-être qu'elle est le lieu par excellence de leur affrontement, et comme le miroir terrible de ce que nous sommes ». (Muno 1985 : 60)

${ }^{14}$ Le thème du quotidien accablant et celui des relations familiales tendues des personnages munoliens ont été étudiés par F. Andriat. Voir : (Andriat 1980 : 5-18).

${ }^{15}$ Ajoutons que la femme insolite munolienne est le caractère inversé de la « femme réelle » (terme employé par V. Tritter, voir : Tritter $2002: 69$ ), soumise aux artifices et aux conventions, étroites et bornées d'une quotidienneté morne. Envahissante et tyrannique, elle emprisonne l'homme dans un petit univers douillet d'où il lui est 
La mort a le visage d'une femme...

Les créatures fantastiques munoliennes sont de toute évidence atypiques, car leur apparition ne provoque pas, pour reprendre le terme freudien, un sentiment $d^{\prime}$ ' inquiétante étrangeté », mais plutôt celui de rassurante familiarité, tant elles sont ordinaires et amicales ${ }^{16}$. Leur surgissement dans la vie des personnages, qui s'opère sous la forme la plus naturelle du monde, comme s'il était régi par les lois de la nature, laisse croire que la mort n'est pas une fin absolue et qu'il existe un autre monde, peut-être pas si redoutable, car il est habité par des êtres bienveillants. Par là, le fantastique de Muno revêt une dimension cathartique évidente: en abordant le thème de la mort qui, sous l'apparence d'une femme attachante, rôde autour des personnages, il l'exorcise et l'apprivoise : de la sorte, le spectre de la Faucheuse devient apaisant malgré son côté inéluctable.

\section{Bibliographie :}

ANDRIAT, Frank (1980), "Jean Muno: la fantaisie du désespoir ", Jean Muno, Bruxelles : Cyclope-DEM, n 28-29-30, 5-18.

BERNARD, Jean-Louis (1971), Les archives de l'insolite, Paris : Le Dauphin.

Chevalier, Jean - Gheerbrant, Antoine (1969), Dictionnaire des symboles, Paris: Laffont.

DenIS, Marie (1989), « Le vampiroloque », in : FrICKX, Robert, Jean Muno, Lausanne : L'Age d'homme, 77-83.

Grivel, Charles (1983), «Le fantastique », in: Mana. Mannheim Analytiques, $\mathrm{n}^{0} 1$, Manenheim : Manenheim Universitat.

Muno, Jean (1979), Les histoires singulières, Bruxelles : Ed. Labor.

Muno, Jean (1985), Les histoires griffues, Lausanne : L'Age d'homme.

MunO, Jean (1989), Extrait d'une lettre envoyée à R. Frickx, in : FRICKX, Robert, Jean Muno, Lausanne : L'Age d'homme, 135-139.

Poulet, Robert (1943), «A propos de Nouvelles réalités fantastiques », in : Partis pris, Bruxelles - Paris : Les Ecrits, 110-111.

Richter, Anne (1989), « Le regard de Jean Muno », in : Frickx, Robert, Jean Muno, Lausanne : L'Age d'homme, 85-88.

SteinMETz, Jean-Luc (1990), Le fantastique, Paris : PUF.

Thyrion, Frank (1986), « Une histoire de tous les jours : L'iguane de Jean Muno », Phénix nº 7, 61-76.

ToDorov, Tzvetan (1970), Introduction à la littérature fantastique, Paris : Seuil.

TRITTER, Valérie (2001), Le fantastique, Paris : Ellipses.

difficile de s'échapper. Dolf Cnorrhinmus, Peter, Walter ou le protagoniste de La dame-au-chien vivent mal la monotonie étouffante de la vie en ménage où, selon F. Andriat, les époux « sont l'image parfaite du couple bougie » et «fendent lentement ensemble sans que rien ne puisse arrêter leur progression vers l'ennui, vers l'absence ou, mieux, vers la fausse présence ». (Andriat 1980:8) Pour fuir cet univers-carcan, ils cherchent un refuge au-delà du monde réel, dans les bras d'une femme étrangère. Ce type de doublet est largement répandu dans la littérature fantastique. La «femme réelle » possède souvent sa réplique supérieure dans le monde onirique auquel aspire le héros : séduisante, imprévisible et dangereuse, la femme fantasmée est l'antithèse de son substitut dans la fade réalité. Voir Tritter $2002: 68,69$.

${ }_{16}$ Par le rejet de l'angoisse ainsi que par l'absence d'ambiguïté et d'hésitation concernant l'interprétation du surnaturel, les nouvelles de Muno s'apparentent au "nouveau fantastique ». Au XXe siècle, les vampires et les fantômes n'effrayent plus personne. Le nouveau fantastique est plutôt " manifestation des fantasmes, d'inquiétudes, de dérèglements intérieurs »; c'est " la contemplation de sa propre existence, menacée dans sa rationalité », écrivent P. Yerlès et M. Lits (Yerles, Lits 1992 : 30). Selon R. Poulet le nouveau fantastique est « celui qui, pour surprendre le secret d'une vie inconnue, s'évertue à pénétrer au coeur du réel, mais sans en troubler le cours, sans en transgresser les lois ». (Poulet $1943: 110$ ) 
Renata Bizek-Tatara

TRITTER, Valérie - Brunel, Pierre (2010), Encyclopédie du fantastique, Paris : Ellipses.

YerLes, Pierre - Lits, Marc (1990), Le fantastique, Bruxelles : Didier Hatier.

Renata Bizek-Tatara

Instytut Filologii Romańskiej

Wydział Humanistyczny

Uniwersytet Marii Curie-Skłodowskiej

Plac M. Curie-Skłodowskiej 4 A

20-031 Lublin

Pologne

rebita@poczta.onet.pl 